Submitted to ApJ

Preprint typeset using $\mathrm{LT}_{\mathrm{E}} \mathrm{X}$ style emulateapj v. 9/08/03

\title{
MAGNETIC DRIVING OF RELATIVISTIC OUTFLOWS IN ACTIVE GALACTIC NUCLEI. I. INTERPRETATION OF PARSEC-SCALE ACCELERATIONS
}

\author{
NeKTARIOS Vlahakis \\ Section of Astrophysics, Astronomy \& Mechanics, Department of Physics, University of Athens, 15784 Zografos Athens, Greece \\ vlahakis@phys.uoa.gr \\ AND \\ ARIEH KÖNIGL \\ Department of Astronomy \& Astrophysics and Enrico Fermi Institute, University of Chicago, 5640 S. Ellis Ave., Chicago, IL 60637 \\ arieh@jets.uchicago.edu \\ Submitted to ApJ
}

\begin{abstract}
There is growing evidence that relativistic jets in active galactic nuclei undergo extended (parsec-scale) acceleration. We argue that, contrary to some suggestions in the literature, this acceleration cannot be purely hydrodynamic. Using exact semianalytic solutions of the relativistic MHD equations, we demonstrate that the parsec-scale acceleration to relativistic speeds inferred in sources like the radio galaxy NGC 6251 and the quasar 3C 345 can be attributed to magnetic driving. Additional observational implications of this model will be explored in future papers in this series.

Subject headings: galaxies: active — galaxies: individual (NGC 6251) — galaxies: jets — ISM: jets and outflows - MHD — quasars: individual (3C 345)
\end{abstract}

\section{INTRODUCTION}

Magnetic acceleration and collimation has long been thought to be the underlying mechanism responsible for the similar manifestations of cosmic jets in such diverse systems as young stellar objects and active galactic nuclei (AGNs) (e.g., Königl 1986; Pringle 1993; Spruit 1996; Livio 2000). Although some AGN jets have long been known to exhibit apparent superluminal motions, with inferred (terminal) bulk Lorentz factors in the blazar class of sources of $\gamma_{\infty} \lesssim 10$ (but exceeding 40 in some cases; e.g., Jorstad et al. 2001), most models to date have concentrated on the nonrelativistic regime. However, two recent discoveries the detection of apparent superluminal motions in certain Galactic black-hole binaries (the so-called microquasars; e.g., Mirabel \& Rodríguez 1999), from which mildly relativistic bulk velocities have been deduced, and the inferred association of gamma-ray bursts (GRBs) with ultrarelativistic $\left(\gamma_{\infty} \gtrsim\right.$ $10^{2}$ ), highly collimated outflows (e.g., Piran 1999) - have highlighted the strong similarities among the various types of relativistic jet sources (e.g., Ghisellini \& Celotti 2002) and have refocused attention on the question of their origin. Although the interpretation of relativistic outflows and the generalization of magnetohydrodynamics (MHD) to the relativistic regime present several distinct challenges, the prevailing view has been that magnetic driving is the common underlying mechanism also in this case (e.g., Blandford 2002).

However, this interpretation of relativistic jets is by no means universal. One example is provided by the radio galaxy NGC 6251, in which Sudou et al. (2000) inferred a bulk acceleration from $V \approx 0.13 c$ to $V \approx 0.42 c$ on sub-parsec scales. This behavior was attributed by Melia. Liu. \& Fatuzzo (2002) to a thermal acceleration of a proton-electron plasma that is heated to a temperature $T \approx 10^{12} \mathrm{~K}$ in a region of radius $r \lesssim 0.03 \mathrm{pc}$. We note, however, that a thermally driven, purely hydrodynamic flow typically undergoes the bulk of its acceleration over a distance that is of the order of the size of the mass distribution that initially confines it by its gravity, which in this case is much smaller than the radius of the ap- parent acceleration zone (see $\S 2$. Centrifugal driving (e.g., Blandford \& Pavne 1982) - the commonly invoked hydromagnetic acceleration mechanism for nonrelativistic jets typically also acts fairly rapidly and thus would similarly fail to account for the large-scale acceleration inferred in NGC 6251. A possible resolution of this puzzle is provided by the finding of Li. Chiueh. \& Begelman (1992), who were the first to generalize the "cold" radially self-similar MHD flows of Blandford \& Pavne (1982) to the relativistic regime (see also Contopoulos 1994), that their solutions contain an extended magnetic pressure-gradient acceleration region beyond the classical fast-magnetosonic point (a singular point of the Bernoulli equation), a behavior that they ascribed to the action of a "magnetic nozzle." It was subsequently shown by Vlahakis et al. (2000) that a similar mechanism operates also in nonrelativistic flows, but the effect is probably easier to discern observationally in relativistic jets.

Vlahakis \& Königl (2003a, hereafter VK) carried out a further generalization by deriving "hot," radially self-similar, relativistic MHD solutions for trans-Alfvénic flows. ${ }^{1}$ They showed that the magnetic field always guides and collimates the flow, but that, if the specific enthalpy $\xi c^{2}$ is initially (subscript $i$ ) $\gg c^{2}$, then an extended thermal acceleration region can develop, within which the flow is accelerated from $\gamma_{i} \approx 1$ to $\gamma \approx \xi_{i}$. If the total energy-to-mass flux ratio $\mu c^{2}$ is $\gg \xi_{i} c^{2}$, corresponding to a Poynting flux-dominated outflow, then the bulk of the acceleration is magnetic and takes place downstream from this point. VK demonstrated that the flow continues to be accelerated all the way up to the modified fast-magnetosonic surface, which is the locus of the fast-magnetosonic singular points of the combined Bernoulli

\footnotetext{
${ }^{1}$ The trans-Alfvénic solutions correspond to a dominant poloidal magnetic field at the base of the flow. Vlahakis \& König] (2003b) derived analogous solutions for super-Alfvénic jets, for which the magnetic field at the base of the flow is predominantly azimuthal. The latter configuration may be expected to apply to inherently nonsteady outflows (e.g., Contopoulos 1995). In this paper we adopt the poloidal field configuration as the most appropriate modeling framework for AGN jets, as has also been done by other workers (e.g., Livio. Pringle, \& King 2003).
} 
and transfield equations and represents the true "causality surface" (or "event horizon") for the propagation of fast waves. VK showed that this singular surface can lie well beyond the classical fast-magnetosonic surface and argued that this is the essence of the "magnetic nozzle" effect.

This is the first in a series of papers in which we apply the $\mathrm{VK}$ formalism to the interpretation of relativistic jets in AGNs. $^{2}$ Our aim is to model a variety of observational findings and attempt to construct basic diagnostic tools for the study of such jets. In this paper we focus on the extendedacceleration signature of magnetically driven jets and use our solutions to model the parsec-scale accelerations already indicated in a number of relativistic jet sources. We first present arguments for why the extended acceleration is unlikely to have a thermal origin $(\$ 2)$. We then consider magnetic jet models: after a brief review of the solution methodology $(\S 3$, we demonstrate $(\S 4$ that magnetic driving can account for Sudou et al.'s observations of NGC 6251 as well for the parsec-scale acceleration to $\gamma_{\infty} \geq 10$ inferred in superluminal blazar jets like 3C 345 (Unwin et al. 1997). Our conclusions are given in $\S 5$

\section{CAN THE JETS BE DRIVEN THERMALLY?}

The sub-parsec-scale kinematics of the NGC 6251 jet was deduced by Sudou et al. (2000) after they discovered the counterjet in this powerful radio galaxy. By applying a relativistic beaming model to the measured variation in the jetcounterjet intensity ratio, they inferred that the outflow is accelerated from $\sim 0.13 c$ at $r \approx 0.53$ pc to $\sim 0.42 c$ at $r \approx$ 1.0 pc. $^{3}$ We now argue that, contrary to the suggestion made in Melia et al. (2002), the cause of this acceleration cannot be thermal pressure driving.

Since the inferred speeds are not too close to $c$, it is sufficient to use nonrelativistic hydrodynamics. In the absence of magnetic forces and assuming spherical symmetry for simplicity, the flow can be described as a Parker (1958) wind, in which the radial velocity $V$ is given as a function of the radius $r$ by

$$
\frac{r}{V} \frac{d V}{d r}=\frac{2 C_{\mathrm{s}}^{2}-G M / r}{V^{2}-C_{\mathrm{s}}^{2}} .
$$

Here $M$ is the mass of the central black hole, $G$ is the gravitational constant, and $C_{\mathrm{s}}=\left(2 \Gamma k_{\mathrm{B}} T / m_{p}\right)^{1 / 2}$ is the sound speed of a fully ionized hydrogen gas (with $\Gamma, m_{p}$ and $k_{\mathrm{B}}$ being the adiabatic index, proton mass, and Boltzmann's constant, respectively). In the best-fit model of Melia et al. (2002) (which is consistent with the limits set by the radio observations of Jones et al. 1986), the base temperature (at $r_{i}=0.026 \mathrm{pc}$ ) is $T_{i}=10^{12} \mathrm{~K}$. For these values of $r$ and $T$, and with $M \approx$ $6 \times 10^{8} M_{\odot}$ (Ferrarese \& Ford 1999), we find that $2 C_{\mathrm{s}}^{2}$ is $\gg G M / r$ and hence that the flow at $r_{i}$ would already be supersonic with $V>C_{\mathrm{s}} \approx 0.55 \mathrm{c}$. By the time such a flow reached the scales mapped by Sudou et al. (2000), its velocity would be significantly larger — and its acceleration substantially smaller - than what has been inferred from the observations.

\footnotetext{
2 We have previously concentrated on applications to GRBs; see VK, where analogies among GRBs, AGNs, and microquasars are discussed, as well as Vlahakis \& Königl (2001, 2003b) and Vlahakis, Peng, \& Königl (2003).

3 Using a more accurate determination of the distance to NGC 6251, Melia et al. (2002) took the radial range of the acceleration region to be $0.30-0.57 \mathrm{pc}$. Since the differences from the values adopted by Sudou et al. 2000) have little impact on our arguments, we continue to use the latter in our discussion.
}

Extended acceleration has also been indicated in superluminal blazar jets. For example, VLBI images of the quasar 3C 345 have shown that the jet component speeds increase with separation from the core (e.g., Zensus et al. 1995; Lobanov \& Zensus 1999). In particular, in the case of the C7 component, Unwin et al. (1997) combined a VLBI propermotion measurement with an inference of the Doppler factor from a synchrotron self-Compton calculation to deduce an acceleration from $\gamma \sim 5$ to $\gamma \gtrsim 10$ over a (deprojected) distance range (measured from the core) of $\sim 3-20 \mathrm{pc}$.

Although blazar jets are also sometimes modeled in terms of a purely hydrodynamic acceleration (e.g. Georganopoulos \& Marscher 1998), one can show quite generally that in this case, too, thermal driving alone cannot account for the observations. A variety of arguments (summarized, e.g., in Ghisellini \& Celotti 2002) indicate that protons are the dynamically dominant component in many AGN jets. Energy conservation in such outflows implies $\gamma_{\infty} / \gamma_{i} \approx 1+2[\Gamma /(\Gamma-1)]\left(k_{\mathrm{B}} T_{i} / m_{p} c^{2}\right)$. Even if the initial temperature were as high as $\sim 10^{12} \mathrm{~K}$, the terminal Lorentz factor would still be $\lesssim 2-$ much smaller than the values typically inferred from the observed superluminal motions. An additional argument can be made on the basis of the observed acceleration rate, which in a purely hydrodynamic model is determined by the radial dependence of the external pressure that provides lateral confinement of the jet. In a simplified picture of a one-dimensional pressure distribution that scales with distance $z$ from the origin as $z^{-\alpha}$, the Lorentz factor of an adiabatic, supersonic jet is predicted to increase as $z^{\alpha / 4}$ (e.g., Blandford \& Rees 1974). Applying this picture to the 3C 345 data reported in Unwin et al. (1997), one infers $\alpha \sim 1.5$ on parsec scales. It is, however, unclear how a pressure distribution of this type could arise in a natural way in an AGN.

The most likely alternative in both of these cases is magnetic acceleration, which we consider in the remainder of this paper.

\section{SEMIANALYTIC SOLUTIONS OF THE "HOT" RELATIVISTIC MHD EQUATIONS}

VK considered the full set of special-relativistic MHD equations, allowing both the bulk and the random speeds to be relativistic. They assumed ideal MHD, axisymmetry, no explicit time dependence, and a polytropic equation of state (with the pressure $P$ scaling with the rest-mass density $\rho_{0}$ as $P \propto \rho_{0}^{\Gamma}$, where the adiabatic index is taken to be $5 / 3$ or $4 / 3$ depending, respectively, on whether the pressure is dominated by "cold" protons and electrons or by "hot" electron-positron pairs and radiation). Under these assumptions, the MHD equations can be partially integrated to yield several field-line constants (with the field line being identified by the poloidal magnetic flux function $A$ ): the total specific angular momentum $L(A)$, the field angular velocity $\Omega(A)$, the magnetization parameter $\sigma_{\mathrm{M}}(A)$ (with the mass-to-magnetic flux ratio given by $\left.A \Omega^{2} / \sigma_{\mathrm{M}} c^{3}\right)$, the adiabat $Q(A)=P / \rho_{0}^{\Gamma}$, and the total energyto-mass flux ratio $\mu(A) c^{2}=\xi \gamma c^{2}-(c / 4 \pi)\left(E B_{\phi} / \gamma \rho_{0} V_{p}\right)$ (where $E$ is the electric field amplitude, $B_{\phi}$ is the azimuthal field component, and $V_{p}$ is the poloidal velocity component). For the adopted equation of state, the specific enthalpy is given by $\xi c^{2}=c^{2}+[\Gamma /(\Gamma-1)]\left(P / \rho_{0}\right)$.

The transfield force-balance equation is integrated under the most general ansatz for radial self-similarity [in spherical coordinates $(r, \theta, \phi))]$, in which the shape $r(A, \theta)$ of a poloidal field line is given as a product of a function of $A$ times a func- 
tion of $\theta: r=\mathcal{F}_{1}(A) \mathcal{F}_{2}(\theta)$ (see Vlahakis \& Tsinganos 1998). Such a separation of variables can be effected if $\mathcal{F}_{1}(A) \propto A^{1 / F}$, $L(A) \propto A^{1 / F}, \Omega(A) \propto A^{-1 / F}, Q(A) \propto A^{-(F-2)(\Gamma-1)}$, and if $\mu(A)=$ const and $\sigma_{\mathrm{M}}(A)=$ const. To obtain a solution, it is necessary to specify seven boundary conditions as well as the values of $\Gamma$ and $F$. The parameter $F$ controls the distribution of the poloidal current $I: 2 I / c=\varpi B_{\phi}=A^{1-1 / F} \mathcal{F}(\theta)$ [using cylindrical coordinates $(\varpi, \phi, z)]$. Close to the origin the field is force-free, with $\mathcal{F}(\theta) \approx$ const, which implies $\varpi B_{\phi} \propto A^{1-1 / F}$. For $F>1$, the current $|I|$ is an increasing function of $A$, corresponding to the current-carrying regime (for which the poloidal current density is antiparallel to the field). A solution with $F>1$ should provide a good representation of the conditions near the axis of a highly collimated flow. Conversely, a solution with $F<1$ corresponds to the return-current regime (for which the poloidal current density is parallel to the field) and may be most suitable at larger cylindrical distances. VK showed that, for $F \gtrsim 1$, the modified fast surface is at infinity and an initially Poynting-dominated flow $\left(\mu \gg \xi_{i}\right)$ attains a rough equipartition between the kinetic and Poynting energy fluxes at large distances from the origin. In this case the Lorentz force efficiently collimates the flow, which reaches cylindrical asymptotics. In contrast, for $F<1$, even though the acceleration is more efficient (so more of the Poynting flux is converted into kinetic energy), the collimation is weaker and the flow only reaches conical asymptotics.

The illustrative solutions derived in VK demonstrated that centrifugal driving plays a limited role in the acceleration of relativistic flows - it is only important initially (for as long as $V_{\phi} \gtrsim V_{p}$ ). As already noted in $\S 1$ thermal acceleration can dominate over a more extended zone if the initial enthalpy is relativistic $\left(\xi_{i} \gg 1\right)$. Magnetic acceleration, however, takes over after $\xi$ drops to $\sim 1$ and can remain important well beyond the classical fast-magnetosonic surface (where $\gamma V_{p} \approx\left[\left(B^{2}-E^{2}\right) /\left(4 \pi \rho_{0} \xi\right)\right]^{1 / 2}$ ). If the Poynting flux is initially smaller than the enthalpy flux (corresponding to $\mu \approx \xi_{i}$ ), the acceleration is predominantly thermal (governed by $\xi \gamma \approx \mu$ ) and, for a trans-Alfvénic solution, the magnetic field only acts to guide the flow. The collimation in this case is weak, and the streamlines are asymptotically conical (see also Vlahakis at al. 2003).

\section{MAGNETIC OUTFLOW MODELS FOR JETS IN RADIO GALAXIES AND QUASARS}

\subsection{The Accelerating Jets in the Radio Galaxy NGC 6251}

Figure 11 shows a solution of the steady, axisymmetric, ideal-MHD equations that describes an outflow from a disk around a supermassive black hole. As in VK, the solution was constructed using the $r$ self-similarity formalism but was restricted to extend over a finite radial range. The dimensional parameter values were determined on the basis of the inferred magnitudes of the NGC 6251 black-hole mass and mass outflow rate. ${ }^{4}$ Given that the slow-magnetosonic singular surface arises from the interplay between gravitational and thermal forces and that the relativistic $r$ self-similar model does not incorporate gravity, the solution only covers the super-slow regime of the flow. This regime, however, is the most pertinent one and - even for the high value of $T_{i}$ that we adopt

\footnotetext{
${ }^{4}$ The values of the model parameters and boundary conditions that define the displayed solution are, in the notation of VK: $\Gamma=5 / 3, F=1.0001, z_{c}=$ $0.7 \mathrm{pc}, x_{\mathrm{A}}^{2}=0.87, \sigma_{\mathrm{M}}=3, \xi_{\mathrm{A}}=1.005, \mu=7.8, \theta_{\mathrm{A}}=50^{\circ}$, and $B_{0}{\varpi_{0}^{2-F}}^{2-}=$ $1.1 \times 10^{16} \mathrm{cgs}$.
}

(following Melia et al. 2002) - contains the $V_{p}>0.13 c$ velocity range measured in the Sudou et al. (2000) observations.

Figure 11 depicts the field-line shape. The innermost field line originates from the vicinity of the black hole, at a (cylindrical) distance $\bar{\varpi}_{\text {in }}$ of a few Schwarzschild radii from the center (for a black hole of mass $M$, the Schwarzschild radius is $\left.\simeq 10^{-13}\left(M / M_{\odot}\right) \mathrm{pc}\right)$, whereas the outermost field line originates at $\bar{\sigma}_{\text {out }}=10^{3} \varpi_{\text {in }}$. For this choice of the $\varpi_{\text {out }} / \varpi_{\text {in }}$ ratio and the inferred mass of the NGC 6251 black hole, the massloss rate in the wind is $\simeq 9 \times 10^{21} \mathrm{~g} \mathrm{~s}^{-1}$ (see Fig. 1b), close to the best-fit estimate of Melia et al. (2002).

Figure 17 shows the various force densities in the poloidal direction along the outermost field line as functions of $\varpi / \varpi_{\mathrm{A}}$ (where $\bar{\varpi}_{\mathrm{A}} \equiv(L / \mu \Omega)^{1 / 2}$ is the Alfvén lever arm; note that, in the $r$ self-similar model, $\Phi / \Phi_{\mathrm{A}}$ is solely a function of the polar angle $\theta$ ). Although the thermal pressure gradient is the dominant force density very close to the origin, the magnetic force rapidly takes over. (The centrifugal force, which could in principle also contribute near the origin, is much smaller than the pressure gradient force in this case.) Figure 1 shows that the Lorentz factor (solid line) increases monotonically with distance. Also shown is the product $\xi \gamma$ (dashed line), which demonstrates (in accord with Fig. 1k) that $\xi$ differs from 1 (signaling that thermal effects contribute to the acceleration) only in the immediate vicinity of the origin $\left(\varpi \lesssim 0.35 \varpi_{\mathrm{A}}\right)$. It is seen that, even for very high initial temperatures (Fig. 11), the thermal contribution to the acceleration is negligible, confirming the conclusion of $\S[2$ The upper curve in Figure 11 indicates that, for $\varpi>0.35 \varpi_{\mathrm{A}}$, the Lorentz factor increases due to a decreasing Poynting-to-mass flux ratio: in this range the Poynting flux is converted into matter kineticenergy flux. Asymptotically the Lorentz factor is $\simeq \mu / 2$, or, equivalently, the flow reaches a rough equipartition between Poynting and kinetic energy fluxes. Although the final value of $\gamma$ in the jets in NGC 6251 is not known, our particular choice of value for $\mu$ implies that $\gamma_{\infty} \approx 4$. This estimate is consistent with the values typically inferred for radio-galaxy jets (e.g., Giovannini et al. 2001).

Figure 11 shows the various components of the flow velocity. Although it is not crucial for a qualitative analysis, we note that the solution presented here actually reproduces the velocities inferred by Sudou et al. (2000). Asymptotically $V_{\varpi} \ll V_{z}$, as expected in solutions that exhibit cylindrical collimation (see $\S 3$ ).

The components of the magnetic field along the outermost field line are shown in Figure1g. The poloidal field initially exceeds the azimuthal component (with the two becoming comparable at $\Phi=\bar{\omega}_{\mathrm{A}}$ ), but at larger distances the azimuthal component dominates. The fields scale roughly as $B_{\phi} \propto \sigma^{-1}$ and $B_{z} \propto \bar{\varpi}^{-2}$.

\subsection{The Accelerating Jet in the Quasar $3 C 345$}

We propose that the parsec-scale acceleration inferred by Unwin et al. (1997) in component C7 of the 3C 345 jet is most plausibly interpreted in terms of magnetic driving, and we present in Figure 2 an $r$ self-similar MHD solution describing a proton-electron jet that supports this claim. ${ }^{5}$ In particular, it is seen from Figure 2 that the Lorentz factor increases from $\gamma \approx 5$ at $\Phi / \varpi_{\mathrm{A}} \approx 5.55$ to $\gamma \approx 10$ at $\varpi / \Phi_{\mathrm{A}} \approx 13.31$.

\footnotetext{
5 The parameters/boundary conditions that determine this solution are, in the notation of VK: $\Gamma=5 / 3, F=0.99, z_{c}=0, x_{\mathrm{A}}^{2}=0.987, \sigma_{\mathrm{M}}=8.66, \xi_{\mathrm{A}}=$ 1.0007, $\mu=75, \theta_{\mathrm{A}}=25^{\circ}$, and $B_{0} \varpi_{0}^{2-F}=2.1 \times 10^{17} \mathrm{cgs}$.
} 

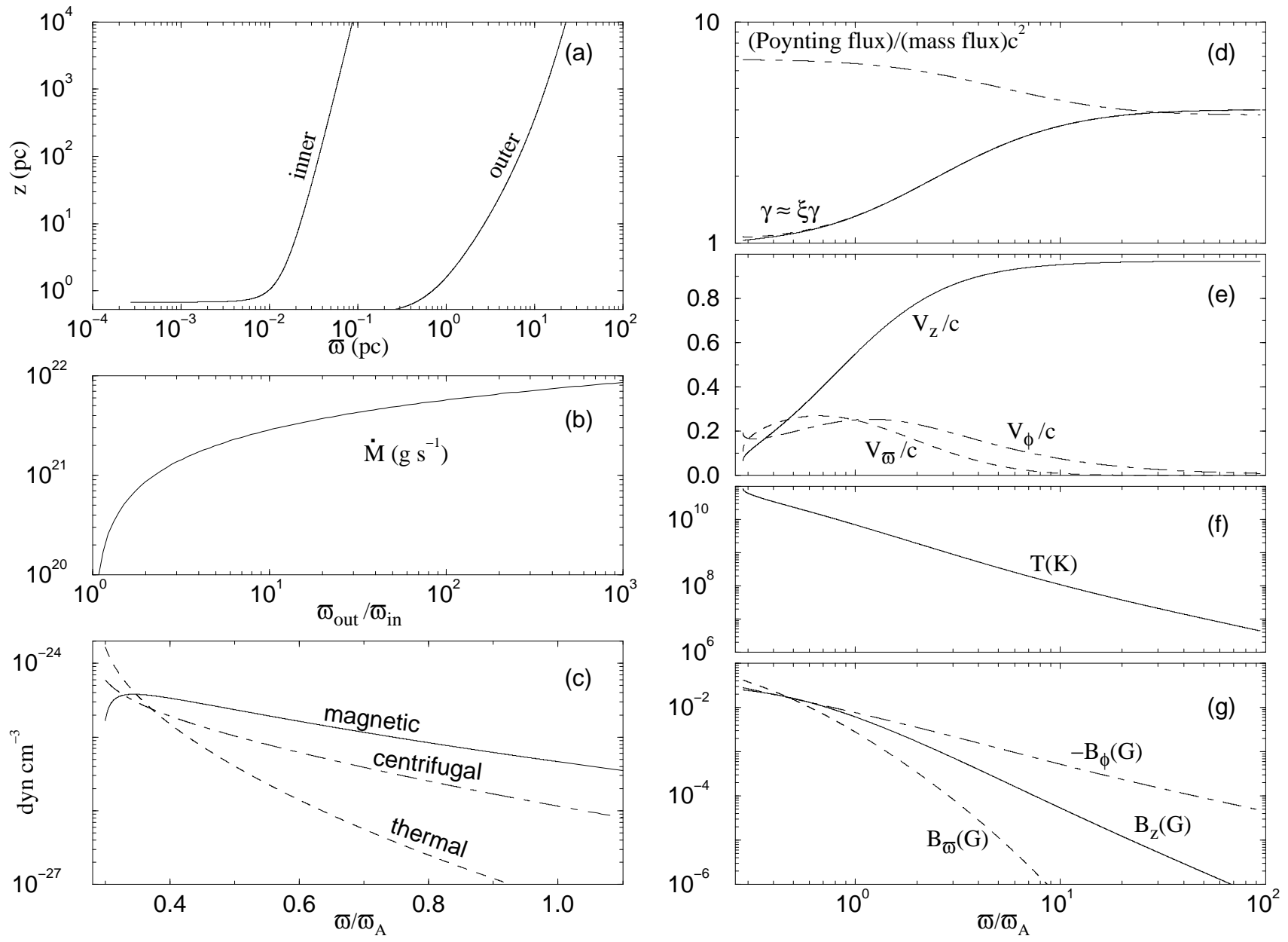

FIG. 1. $-r$ self-similar solution describing the jets in NGC 6251. (a) Poloidal field-line shape on a logarithmic scale. (b) Mass loss rate as a function of $\varpi_{\text {out }} / \varpi_{\text {in }}$, the ratio of the outermost and innermost disk radii. The remaining panels show the force densities in the poloidal direction $(c)$ and various other quantities $\left[(d)-(g)\right.$; see text for details] as functions of $\varpi / \varpi_{\mathrm{A}}$ (which, in turn, is a function of the polar angle $\theta$ ) along the outermost field line. Here $\Phi_{\mathrm{A}}$ is the Alfvén lever arm, which equals $\Phi_{\mathrm{A}, \text { in }}=9.8 \times 10^{-4} \mathrm{pc}$ and $\Phi_{\mathrm{A}, \text { out }}=10^{3} \varpi_{\mathrm{A}, \text { in }}=0.98 \mathrm{pc}$ on the innermost and outermost field lines, respectively. Along the innermost field line, the quantities plotted in panels $(d),(e)$, and $(f)$ remain the same functions of $\varpi / \varpi_{\mathrm{A}}$, whereas the quantities shown in panels $(c)$ and $(g)$ are $\bar{\varpi}_{\mathrm{A}, \text { out }} / \bar{\varpi}_{\mathrm{A}, \text { in }}$ times larger.

Using the value of the Alfvén lever arm on the outer field line $\left(\Phi_{\mathrm{A}, \text { out }}=4.1 \times 10^{-2} \mathrm{pc}\right)$, we find that the cylindrical distance changes from $\Phi \approx 0.23 \mathrm{pc}$ to $\Phi \approx 0.546 \mathrm{pc}$; on the basis of Figure $2 \mathrm{a}$, these cylindrical radii correspond to linear distances from the origin of $\sim 3 \mathrm{pc}$ and $\sim 20 \mathrm{pc}$, respectively, in close correspondence with the observed values.

The quasar jet solution shown in Figure 2 is characterized by a significantly higher value of the total energy-to-mass flux ratio $\mu c^{2}$ than the radio-galaxy solution depicted in Figure 1. The terminal Lorentz factor, which again corresponds to a rough equipartition between the asymptotic Poynting and kinetic energy fluxes $\left(\gamma_{\infty} \approx \mu / 2\right)$ is correspondingly higher. For the adopted fiducial parameters, the $\mathrm{C} 7$ component is predicted to continue accelerating up to $\gamma_{\infty} \approx 35$. Interestingly, Lorentz factors of this order have been inferred in the more distant components (in particular, C3 and C5) of the 3C 345 jet (Lobanov \& Zensus 1999).

In comparison with the radio-galaxy solution of $\S 4.1$ the quasar solution presented in this subsection corresponds to a more massive outflow (with a mass-loss rate $\sim 10^{23} \mathrm{~g} \mathrm{~s}^{-1}$ for $\varpi_{\text {out }} / \Phi_{\text {in }}=150$; see Fig. 2 2 ) and to a stronger magnetic field (cf. Figs. 1, and 2g). We note, however, that the den- sity and magnetic field strength are not uniquely determined from the kinematic data: exactly the same flow speeds and field-line shape are obtained if the density, particle pressure, and squared amplitudes of the magnetic field components are rescaled by the same factor.

\section{SUMMARY AND DISCUSSION}

We have argued that acceleration of AGN jets to relativistic velocities on scales that are much larger than the gravitational radius of the central black hole is most plausibly explained in terms of magnetic driving. This mechanism involves acceleration by the gradient of the azimuthal magnetic-field pressure and is distinct from centrifugal acceleration, which is often considered to be the dominant driving mechanism of nonrelativistic jets. Centrifugal driving takes place in the subAlfvénic flow regime and accelerates the gas to a poloidal speed that is of the order of the initial Keplerian speed in the underlying disk. In comparison, magnetic pressure-gradient acceleration occurs over a much more extended region (up to the modified fast-magnetosonic surface) and can produce a much higher (relativistic) terminal speed depending on the initial Poynting-to-mass flux ratio $\mu c^{2}$. In the trans-Alfvénic 

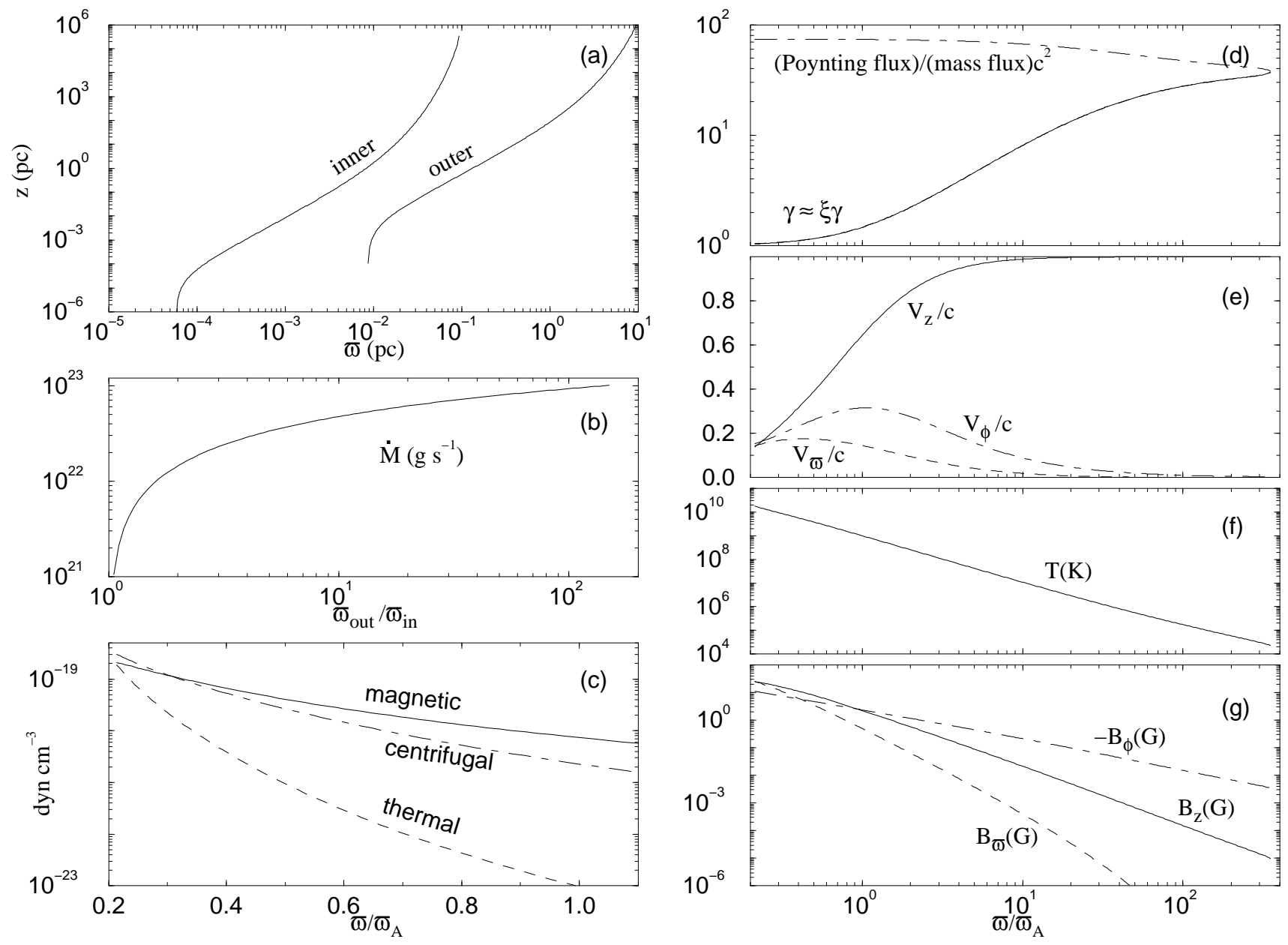

FIG. 2.- Same as Fig. 11 but for the application to the superluminal jet in $3 \mathrm{C} 345$. Here $\varpi_{\mathrm{A}, \text { in }}=2.7 \times 10^{-4} \mathrm{pc}$ and $\varpi_{\mathrm{A}, \text { out }}=150 \varpi_{\mathrm{A}, \text { in }}=4.1 \times 10^{-2} \mathrm{pc}$.

relativistic-MHD solutions presented in this paper, the terminal Lorentz factor is $\gamma_{\infty} \approx \mu / 2$, corresponding to a rough equipartition between the asymptotic Poynting and kineticenergy fluxes. ${ }^{6}$ These solutions are also characterized by strong magnetic collimation (with the streamlines tending asymptotically to cylinders) and are thus consistent with the narrow opening angles inferred in AGN jets.

Thermal effects could in principle contribute to the acceleration even in jets where a large-scale magnetic field guides the flow. As discussed in VK, there are in general two thermal force densities: the pressure gradient $-\nabla P$ and the "temperature" force $-\gamma^{2} \rho_{0}(V \cdot \nabla \xi) V=-\left(\gamma^{2} V / c^{2}\right) V \cdot \nabla P$. These forces accelerate the flow to $\gamma \approx \xi_{i}$. In cases where $\xi_{i} \gtrsim 1$, the thermal acceleration takes place in the nonrelativistic regime and is terminated by the time the speed increases to $\sqrt{3} C_{\mathrm{s}, i}-$ i.e., just beyond the sonic surface that typically lies very close to the origin. As pointed out in $\S[2$ this situation applies to proton-electron outflows; therefore, to the extent that AGN jets have a dynamically dominant proton component (as is often inferred to be the case), their acceleration to relativistic speeds will not be significantly influenced by

\footnotetext{
${ }^{6}$ Note that the Lorentz factor on the classical fast-magnetosonic surface is only $\sim \mu^{1 / 3}$ (e.g., Camenzind 1986), so most of the acceleration in these solutions occurs in the super-fast regime.
}

thermal effects. ${ }^{7}$

Although we only considered two specific applications sub-parsec-scale acceleration involving moderately relativistic speeds in a radio-galaxy jet (NGC 6251; $\$ 4.1$ and parsecscale acceleration involving highly relativistic speeds in a superluminal radio quasar $(3 \mathrm{C} 345 ; \S 4.2$ - there are already several other reported cases of relativistic AGN jets that show evidence for a parsec-scale acceleration. In some cases this has been deduced from an increase in the apparent speed of a particular superluminal component (e.g. Hough. Zensus. \& Porcas 1996). In other cases, where there are observations of several superluminal components, it was found that the innermost one typically exhibits the smallest proper motion, with more distant components indicating an acceleration on parsec scales (e.g., Homan et al. 2001). In the case of the quasar 3C 279 jet, Piner et al. (2003) inferred an acceleration from $\gamma=8$ at $r<5.8 \mathrm{pc}$ to $\gamma=13$ at $r \approx 17.4 \mathrm{pc}$ using a similar approach to the one that had been employed by

\footnotetext{
${ }^{7}$ In the case of an electron-positron outflow, or under optically thick conditions when radiation pressure contributes strongly to the specific enthalpy, one can have $\xi_{i} \gg 1$. In this case the thermal driving (dominated by the "temperature" force) acts well beyond the sonic surface and accelerates the flow to a highly relativistic speed (see $\S 1$. If, in addition to $\xi_{i} \gg 1, \mu \gg \xi_{i}$ also holds, then magnetic driving takes over at the end of the thermal acceleration zone and eventually increases the Lorentz factor to $\sim \mu$ (see $\S 3$ ): this is the behavior obtained in the GRB jet models referenced in footnote 2
} 
Unwin et al. (1997) in 3C 345. It is also worth noting in this connection that a variety of observations indirectly support the magnetic acceleration picture for AGN jets. For example, the parsec-scale helical field morphology implied by our model is consistent with VLBI polarization maps of BL Lac objects (e.g., Gabuzda et al. 2000) and with circular-polarization measurements of blazars (e.g., Homan, Attridge, \& Wardle 2001). However, we defer a more detailed discussion of the additional observational implications of this model to future publications in this series.

In conclusion, we reemphasize that our modeling framework is quite general and is potentially applicable to relativistic jets in a variety of astrophysical settings. In our previ- ous application to GRBs, the model could account for the inferred values of $\gamma_{\infty}$ and of the upper limit $\left(\sim 10^{14} \mathrm{~cm}\right)$ on the size of the acceleration region, but it could not be further constrained because the acceleration region in GRB sources is not resolved. In contrast, the motion of the radio components in certain microquasar jets has been monitored on scales $\sim 10^{16} \mathrm{~cm}$ (e.g., Fender et al. 1999). It would thus be interesting to search for evidence of extended acceleration in these sources, in analogy with the situation in AGN jets.

This work was supported in part by NASA grants NAG59063 and NAG5-12635.

\section{REFERENCES}

Blandford, R. 2002, in Relativistic Flows in Astrophysics, ed. A. W. Guthmann et al. (Lecture Notes in Physics 589; Berlin: Springer), 227

Blandford, R. D., \& Payne, D. G. 1982, MNRAS, 199, 883

Blandford, R. D., \& Rees, M. J. 1974, MNRAS, 169, 395

Camenzind, M. 1986, A\&A, 162, 32

Contopoulos, J. 1994, ApJ, 432, 508

Contopoulos, J. 1995, ApJ, 450, 616

Fender, R. P., et al. 1999, MNRAS, 304, 865

Ferrarese, L, \& Ford, H. C. 1999, ApJ, 515, 583

Gabuzda, D. C., Pushkarev, A. B., \& Cawthorne, T. V. 2000, MNRAS, 319, 1109

Georganopoulos, M., \& Marscher, A. P. 1998, ApJ, 506, 621

Ghisellini, G., \& Celotti, A. 2002, in Blazar Astrophysics with BeppoSAX and Other Observatories, ed. P. Giommi, E. Massaro, \& G. Palumbo (Frascati: ASI Science Data Center, ESA-ESRIN), 257 (astro-ph/0204333 |

Giovannini, G., Cotton, W. D., Feretti, L., Lara, L., \& Venturi, T. 2001, ApJ, 552,508

Homan, D. C., Attridge, J. M., \& Wardle, J. F. C. 2001, ApJ, 556, 113

Homan, D. C., Ojha, R., Wardle, J. F. C., Roberts, D. H., Aller, M. F., Aler, H. D., \& Hughes, P. A. 2001, ApJ, 549, 840

Hough, D. H., Zensus, J. A., \& Porcas, R. W. 1996, ApJ, 464, 715

Jones, D. L., et al. 1986, ApJ, 305, 684

Jorstad, S. G., Marscher, A. P., Mattox, J. R., Wehrle, A. E., Bloom, S. D., \& Yurchenko, A. V. 2001, ApJS, 134, 181

Königl, A. 1986, Ann. NY Acad. Sci., 470, 88

Li, Z.-Y., Chiueh, T., \& Begelman, M.C. 1992, ApJ, 394, 459

Livio, M. 2000, in AIP Conf. 522, Cosmic Explosions, ed. S. S. Holt, \& W. W. Zhang (Melville: AIP), 275
Livio, M., Pringle, J. E., \& King, A. R. 2003, ApJ, 593, 184

Lobanov, A. P. \& Zensus J. A. 1999, ApJ, 521, 509

Melia, F., Liu, S., \& Fatuzzo, M. 2002, ApJ, 567, 811

Mirabel. I. F., \& Rodríguez, L. F. 1999, ARA\&A, 37, 409

Parker, E. N. 1958, ApJ, 128, 664

Piner, B. G., Unwin, S. C., Wehrle, A. E., Zook, A. C., Urry, C. M., \& Gilmore, D. M. 2003, ApJ, 588, 716

Piran, T. 1999, Phys. Rep., 314, 575

Pringle, J. E. 1993, in Astrophysical Jets, ed. M. Burgerela, M. Livio, \& C. O’Dea (Cambridge: Cambridge Univ. Press), 1

Spruit, H. C. 1996, in Evolutionary Processes in Binary Stars, ed. R. A. M. J. Wijers, M. B. Davies, \& C. A. Tout (NATO ASI Ser. C, 477: Dordrecht: Kluwer), 249

Sudou, H., et al. 2000, PASJ, 52, 989

Unwin, S. C., Wehrle, A. E., Lobanov, A. P., Zensus J. A., Madejski, G. M., Aller, M. F., \& Aller, H. D. 1997, ApJ, 480, 596

Vlahakis, N., \& Königl, A. 2001, ApJ, 563, L129

Vlahakis, N., \& Königl, A. 2003a, ApJ, 596, 1080 (VK)

Vlahakis, N., \& Königl, A. 2003b, ApJ, 596, 1104

Vlahakis, N., Peng, F., \& Königl, A. 2003, ApJ, 594, L23

Vlahakis, N., \& Tsinganos, K. 1998, MNRAS, 298, 777

Vlahakis, N., Tsinganos, K., Sauty, C., \& Trussoni, E. 2000, MNRAS, 318, 417

Zensus J. A., Cohen, M. H., \& Unwin, S. C. 1995, ApJ, 443, 35 